\title{
Angiogenic Activity of Sera From Interstitial Lung Disease Patients in Relation to Clinical and Radiological Changes
}

\author{
T. M. Zielonka ${ }^{1}$, U. Demkow 2 , K. Zycinska ${ }^{1}$, M. Filewska 3 , B. Bialas ${ }^{3}$, J. Kus³, E. Radzikowska ${ }^{3}$, \\ P. Remiszewski ${ }^{3}$, J. Szopinski ${ }^{3}$, A. Soszka ${ }^{3}$, K. A. Wardyn ${ }^{1}$, E. Skopinska-Rozewska ${ }^{4}$ \\ ${ }^{1}$ Department of Family Medicine and ${ }^{2}$ Department of Laboratory Diagnostics and Clinical Immunology of Developmental Age, Warsaw \\ Medical University, Warsaw, Poland; ${ }^{3}$ Institute of Tuberculosis and Lung Diseases, Warsaw, Poland; \\ ${ }^{4}$ Department of Pathology, Biostructure Center, Warsaw Medical University, Warsaw, Poland
}

\begin{abstract}
Objective: Clinical symptoms and radiological changes are useful in monitoring patients with interstitial lung diseases (ILD). Neovascularization participates in the pathogenesis of idiopathic pulmonary fibrosis and other ILD. The objective of the study was to examine the relationships between angiogenic activity of sera from ILD patients and clinical or radiological status. Material and methods: Serum samples were obtained from 83 patients with sarcoidosis, 31 with idiopathic pulmonary fibrosis (IPF), 29 with hypersensitivity pneumonitis (HP), 16 with collagen diseases with pulmonary manifestation (CD), 13 with scleroderma (SCL), 14 with Wegener's granulomatosis (WG), 12 with pulmonary Langerhans cell histiocytosis (HIS), 12 with pneumoconiosis (PNC), 10 with drug-induced lung disease (DLD), 5 with cryptogenic organizing pneumonia (COP), and from 36 healthy volunteers. As an angiogenic test we used a cutaneous angiogenesis assay according to Sidky and Auerbach. Clinical status was evaluated using a special questionnaire. In all patients chest radiographs were performed.

Results: The angiogenic properties of sera from ILD differed depending on the clinical diagnosis. The strongest proangiogenic effect was induced by sera from patients with HP (mean number of new vessels 16.8), CD (16.6), sarcoidosis (16.3), IPF (16.2), and PNC (15.7). In the case of DLD (13.2), the effect was comparable to healthy controls (13.5). In contrast, sera from SCL (mean number of the vessels 10.5) and HIS patients (10.8) significantly inhibited angiogenesis compared with controls. The angiogenic activity of sera from patients with hilar or mediastinal lymph nodes involvement was higher than that of sera from patients with lung fibrosis. There were also differences in the serum angiogenic activity in relation to the severity of dyspnea.

Conclusions: The data showed that sera from ILD patients constitute a source of mediators modulating angiogenesis, but the pattern of reaction is different in various diseases. Sera from HP, sarcoidosis, IPF, and $\mathrm{CD}$ patients demonstrated the strongest proangiogenic activity. However, sera from SCL and HIS inhibit angiogenesis. Angiogenic activity of examined sera was related to the clinical and radiological changes.
\end{abstract}

Key words: angiogenesis, interstitial lung diseases, clinical symptoms, radiological changes

\section{INTRODUCTION}

Angiogenesis, a process of new capillary formation from pre-existing vessels, is an important biological event occurring in many physiological and pathological processes, such as an embryonic development, formation of an inflammatory granulation during wound healing, chronic inflammation, and the growth of malignant solid tumors [1-4]. Vessel formation is a multistep process which requires endothelial cell migration, proliferation and degradation of extracellular matrix, and capillary tube formation which is mediated by various angiogenic factors such as vascular endothelial growth factor (VEGF), basic fibroblast growth factor (bFGF), interleukin 8 (IL-8), and others [5]. In contrast, the precise regulation of angiogenesis during wound healing, tumor angiogenesis is characterized by an imbalance between pro-angiogenic and antiangiogenic growth factors and cytokines [6]. Chronic fibroproliferative disorders such as pulmonary fibrosis are also associated with aberrant angiogenesis [7]. Idiopathic pulmonary fibrosis and fibrosing alveolitis associated with systemic sclerosis are characterized by a substantial vascular redistribution, with a shift of interstitial vessels away from airspaces [7]. Vascular remodeling was observed in the fibrotic areas of the lungs but the relevance of these findings in relation to the pathophysiology of fibrosing alveolitis requires further investigation. Other interstitial lung diseases (ILD) characterized by inflammation, cell proliferation, excessive extracellular matrix deposition and several cytokines, are also characterized by aberrant angiogenesis, playing an important role in their pathogenesis [8]. CXC chemokines such as IL-8 and epithelial neutrophil activating protein 78 are involved in the control of the angiogenesis [9]. Previously, we demonstrated that serum angiogenic activity in a number of ILD, such as sarcoidosis, pulmonary fibrosis, and silicosis, is enhanced [10-12].

Although our knowledge on mechanisms of neovascularization on the cellular level has been constantly increasing, little is known to what extend neovascu- 
larization in ILD is a harmful process or is involved in the regeneration of the damaged structures. The effect of angiogenesis on the development of clinical symptoms and radiological changes in patients with ILD has not yet been determined. Therapeutic goals in diffuse lung disease include improvements in the clinical, functional and radiological abnormalities and slowing or preventing of a decline of lung function [13]. The aim of the present study was to evaluate the angiogenic activity of ILD patients' sera in relation to clinical symptoms and radiological changes.

\section{Material AND Methods}

The protocol of the study was approved by a local Ethics Committee. The study population consisted of 225 patients with ILD and 36 healthy volunteers (HV) aged from 18 to 77 years. There were 83 patients with sarcoidosis, 31 with idiopathic pulmonary fibrosis (IPF), 29 with hypersensitivity pneumonitis (HP), 16 with pulmonary manifestation of collagen diseases (CD), 13 with scleroderma (SCL), 14 with Wegener's granulomatosis (WG), 12 with pulmonary Langerhans cell histiocytosis (HIS), 12 with pneumoconiosis (PNC), 10 with drug-induced lung disease (DLD), and 5 with cryptogenic organizing pneumonia (COP). A hundred and ninety of the enrolled subjects never smoked tobacco.

The study population consisted of 22 patients with sarcoidosis in radiographic stage I, 38 patients in stage II, and 23 patients in stage III (ATS/ERS/WASOG classification). In this group there were 35 women and 48 men aged $40 \pm 12$ years. The diagnosis of sarcoidosis was based on clinical, radiological and pathological findings according to ATS/ERS/WASOG recommendation [14]. Fourteen women and 17 men aged $61 \pm$ 12 years with IPF were examined. A diagnosis of IPF was based on clinical, radiological and pathological findings, according to ATS/ERS standards [15]. Sixteen women and 13 men with HP aged $47 \pm 16$ years were evaluated. The diagnosis of HP was based on clinical, radiological, functional, serological, BAL and pathological findings according to the described criteria [16]. The majority of them suffered from avian fancier's lung and farmer's lung. Eleven women and 2 men with SCL (mean age $51 \pm 12$ years) and 7 women and 9 men with other CD (mean age $55 \pm 11$ years) were enrolled. In all patients with collagen diseases (scleroderma, rheumatoid arthritis, systemic lupus erythematosus, dermatomyositis, mixed connective tissue disease and psoriatic arthritis) pulmonary interstitial manifestations were confirmed by high resolution computed tomography. In the study group there were 12 women and 2 men with WG, aged $49 \pm 18$. All patients fulfilled the diagnostic criteria of the American Rheumatism Association for appropriate diseases. The group of patients with pneumoconiosis consisted of eleven men and one woman (mean age $43 \pm 96$ years). In all the cases diagnosis of silicosis was established. In 4 women and 8 men, aged $37 \pm 13$, the diagnosis of HIS was based on clinical, radiological and pathological criteria. All patients with HIS were smokers. In ten patients ( 3 women and 7 men; mean age $63 \pm 11$ years) the diagnosis of a drug-induced lung disease provoked by amiodarone, nitrofurantoin, colchicine, methotrexate, cyclophosphamide, and gold salts was established. The diagnosis of COP according to ATS/ERS standards [17] was established in 3 women and 2 men (mean age $58 \pm 8$ years). Sera from 36 healthy volunteers (22 women and 14 men, aged $39 \pm$ 11) recruited from medical staff were used as a control. Serum was obtained by centrifugation, aliquoted and stored at $-70^{\circ} \mathrm{C}$.

General symptoms (such as weakness, arthralgia, fever $>37.5^{\circ} \mathrm{C}$ ) as well as pulmonary symptoms including cough, and dyspnea were evaluated in all patients using a special questionnaire. Dyspnea was divided into 5 steps: 0 - without dyspnea, 1 - mild dyspnea after big effort, 2 moderate dyspnea, 3 severe dyspnea, 4 very severe at rest.

In all patients standard postero-anterior and lateral chest radiographs by AMBER (Advanced Multiple Beam Equalization Radiography) were obtained. There were five types of radiological changes: 0 - normal chest X ray, 1 - ground glass, 2 - micronodular changes, 3 - fibrotic changes, 4 - enlargement of hilar or mediastinal lymph nodes.

To assess the effect of sera from ILD patients on angiogenesis, leukocytes induced angiogenesis assay in an animal model described by Sidky and Auerbach [18] with modification [11] was used. Normal human peripheral blood mononuclear cells (MNC) derived from the buffy-coat cells of healthy blood donors (Warsaw Central Blood Bank) were prepared using the gradient technique. The samples were diluted 1:1 with PBS, layered over Histopaque 1077 (Sigma) and centrifuged for $10 \mathrm{~min}$ at $500 \mathrm{~g}$ at a room temperature. Female inbred 8 week-old Balb/c mice served as recipients of MNC preincubated in PBS supplemented with 25\% of serum from ILD patients or from healthy volunteers for one hour at $37^{\circ} \mathrm{C}$. As a control, MNC were also preincubated only in PBS. The samples of $0.05 \mathrm{ml} \mathrm{vol}$ ume containing $5 \times 10^{5} \mathrm{MNC}$ in Parker medium supplemented with $0.01 \%$ trypan blue were injected intradermally into anesthetized with $3.6 \%$ chloral hydrate mice. Three days after the cells injection the mice were sacrificed. New blood vessels localized to the injection sites, trypan blue negative and contrasting with the pre-existing vasculature by a virtue of their tortuosity and divarications were counted in a dissection microscope (Nikon, primary magnification 6x). The result was evaluated blindly by the same person based on the previously described criteria by Sidky and Auerbach [18]. The results are introduced as the mean number of new blood vessels grown in one group (18 injection points per patient).

Statistical evaluation of the results was performed by Student's t-test and Pearson's correlation (Statistica 6 for Windows). The data was presented as mean \pm SD and $\mathrm{P}<0.05$ was regarded as the statistical significance. For standardization of the value of the angiogenesis test, the results were averaged and expressed as a number of new vessels and an angiogenesis index (AI). AI represented the mean number of new blood vessels after the injection of MNC preincubated with serum of ILD patients divided by the mean number of new blood vessels after the injection of MNC preincubated with serum of healthy volunteers. 


\section{RESULTS}

\section{Angiogenic Activity}

Sera from the healthy volunteers stimulated angiogenesis compared with PBS (Fig. 1). The mean number of new vessels induced by normal MNC preincubated with sera from the healthy controls $13.7 \pm 0.9$ was significantly $(\mathrm{P}<0.001)$ higher compared with the mean number of new vessels for MNC preincubated with PBS $12.0 \pm 1.2$. Similar angiogenic activity to that of sera from the healthy donors was demonstrated after preincubation of MNC with sera from DLD patients $(13.2 \pm 1.4)$. However, sera from HIS and SCL patients presented antiangiogenic properties compared with healthy donor's sera and to PBS. The number of new vessels created after injection of MNC preincubated with sera from HIS patients $(10.8 \pm 0.5)$ was similar to the number of vessels after preincubation with sera from SCL patients $(10.4 \pm 0.7)$. Incubation of MNC with sera from other ILD significantly stimulated angiogenesis compared with the healthy donors. The number of newly created vessels in this model was $15.4 \pm 1.0$ for COP, $15.7 \pm 0.2$ for pneumoconiosis, $16.2 \pm 0.9$ for IPF, $16.3 \pm 1.6$ for sarcoidosis, 16.6 \pm 1.6 for $\mathrm{CD}, 16.9 \pm 1.4$ for $\mathrm{WG}$, and $17.5 \pm 1.3$ for HP. There were significant differences between the numbers of new vessels created after incubation of sera from different groups (Fig. 1).

\section{Angiogenic Activity of Sera in ReLation to CliniCAL SyMPTOMS}

No correlation between the number of new vessels and the patients' age was observed. The mean number
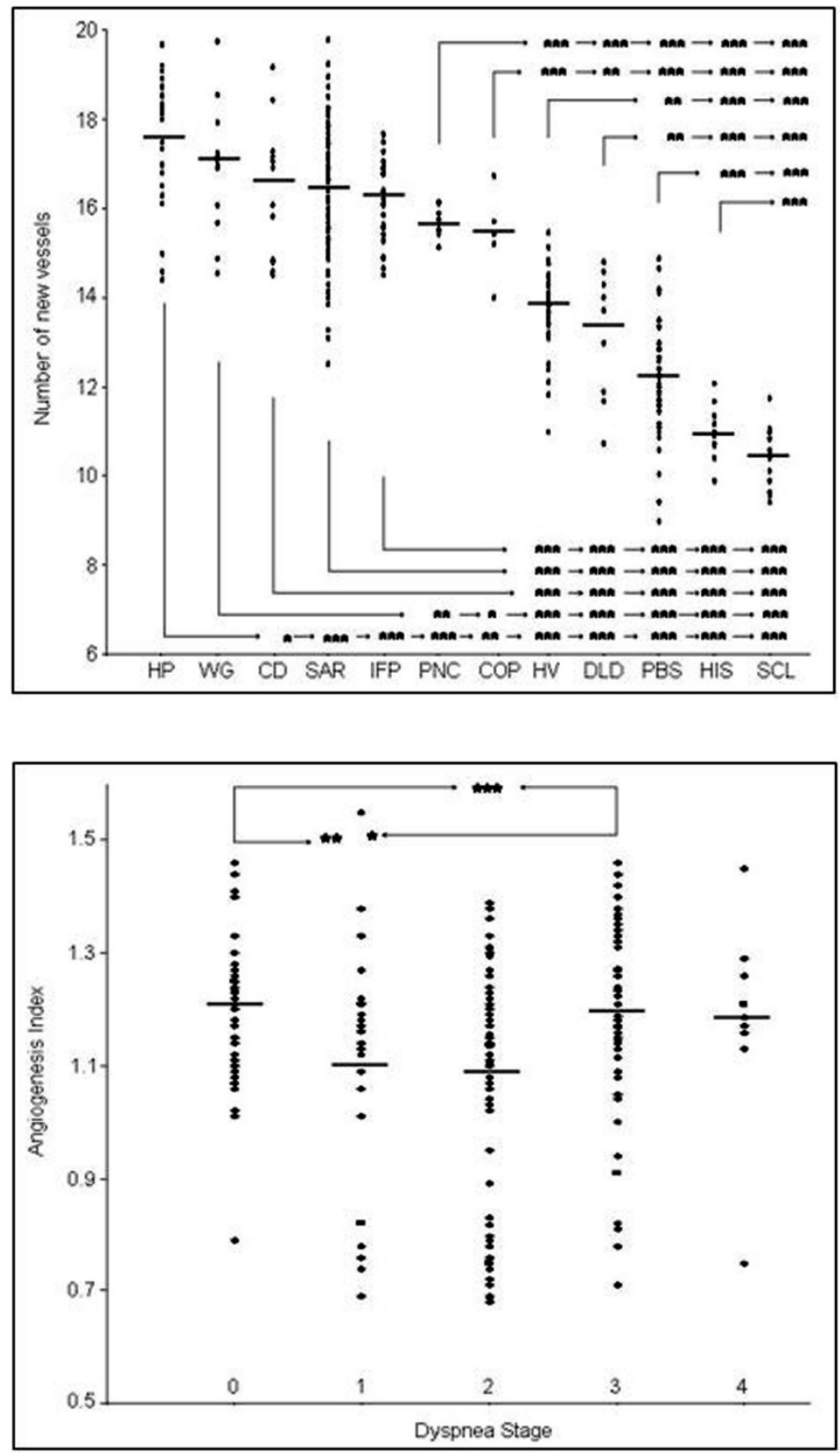

Fig. 1. Number of new vessels created after injection of MNC preincubated in sera from HP patients $(n=29)$, WG patients $(n=14), C D$ patients $(\mathrm{n}=16)$, SAR patients (83), IPF patients $(\mathrm{n}=31)$, PNC patients $(\mathrm{n}=12), \mathrm{COP}$ patients $(\mathrm{n}=5)$, DLD patients $(\mathrm{n}=10)$, HIS patients (12), SCL patients (13), and healthy volunteers (HV) $(n=36)$ or PBS $(n=36)$. The mean values are indicated by horizontal bars, significant differences between the groups are as follows: $* \mathrm{P}<0.05$, $* * \mathrm{P}<0.01$, and $* * * \mathrm{P}<0.001$.
Fig. 2. Angiogenesis index in relation to step of dyspnea: 0 - without dyspnea $(n=50), 1$ - mild dyspnea after big effort $(\mathrm{n}=30), 2$ moderate dyspnea $(n=81), 3$ - severe dyspnea $(n=55), 4$ - very severe at rest $(n=9)$. The mean values are indicated by horizontal bars, significant differences between the groups are as follows: $* \mathrm{P}<0.05$, $* * \mathrm{P}<0.01$, and $* * * \mathrm{P}<0.001)$. 


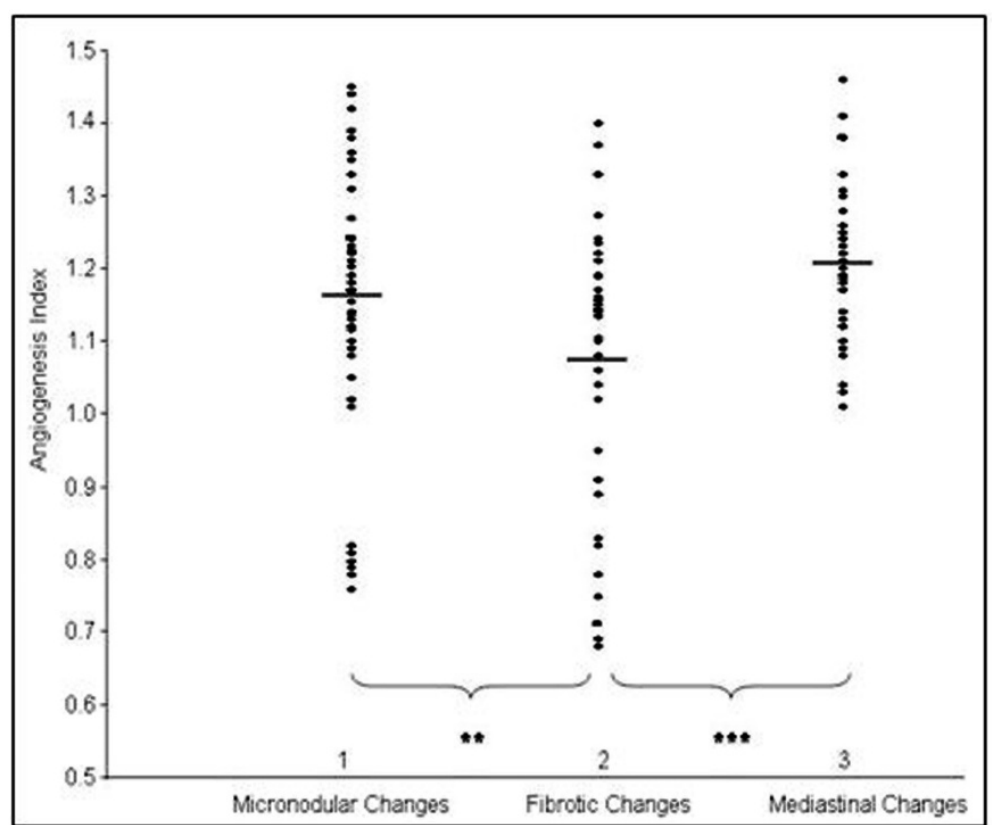

Fig. 3. Angiogenesis index in relation to radiological changes: 1 - micronodular changes $(\mathrm{n}=$ 46), 2 - fibrotic changes $(n=33)$, and 3 - mediastinal changes $(n=32)$. The mean values are indicated by horizontal bars, significant differences between the groups are as follows: $* * \mathrm{P}<0.01$ and $* * * \mathrm{P}<0.001$. of new vessels in the group of men $16.0 \pm 2.0$ $(n=119)$ was similar to the number of vessels in the group of women $15.6 \pm 2.3(n=106)$. The number of new vessels in the group of 67 smokers was $15.4 \pm 2.5$ (AI $1.13 \pm 0.19)$ and for 158 nonsmokers $15.8 \pm 2.3$ (AI $1.2 \pm 0.2$ ). The mean number of new vessels in the group of patients with the presence of general clinical symptoms $(15.8 \pm 2.35)$ was similar to the number of new vessels in the group of asymptomatic patients $(15.4 \pm 2.37)$. The differences observed between AI in the group of symptomatic $(1.16 \pm 0.18)$ and asymptomatic $(1.1 \pm 0.2)$ patients were not significant. No difference was observed in the angiogenic properties of sera from 68 coughing patients $15.7 \pm$ 2.4 (AI $1.2 \pm 0.2$ ) and 157 non-coughing patients 15.6 \pm 2.4 (AI $1.1 \pm 0.2$ ). There were some differences observed between the angiogenic activity of sera from patients with and without dyspnea; dyspnea stage 0 $16.3 \pm 1.6(\mathrm{AI}-1.2 \pm 0.1), 1-15.3 \pm 2.5$ (AI - $1.1 \pm$ 0.2 ), $2-15.1 \pm 2.7$ (AI - $1.1 \pm 0.2), 3-15.9 \pm 2.2$ (AI $1.2 \pm 0.2$ ), and $4-16.6 \pm 2.2$ (AI - $1.2 \pm 0.2$ ) (Fig. 2).

\section{Angiogenic ACtivity of Sera in Relation to RADIOLOGICAL STATUS}

In the present study, only the patients with clear picture of fibrotic, micronodular, or mediastinal radiological changes were included into the analysis. The lowest number of new vessels was observed in the group of 33 patients with fibrotic changes $14.6 \pm 2.5$ (AI $1.08 \pm 0.18)$ compared with 32 patients with enlargement of mediastinal lymphatic nodes $-16.8 \pm 1.5$ (AI $-1.2 \pm 0.1)$, and to 46 patients with micronodular changes - $15.9 \pm 2.0$ (AI - $1.2 \pm 0.2$ ). Differences between these groups were significant (Fig. 3).

\section{Discussion}

Interstitial lung diseases constitute a big group of disorders of various etiology and pathogenesis [19]. Inflammation and pulmonary fibrosis are the most im- portant changes in the course of ILD. Cough and dyspnea are the main clinical symptoms specific for ILD patients. Dyspnea after effort provoked by disturbances of gas exchanges and hypoxia is typical for the advanced stages of the disease. Chronic inflammation and hypoxia are related to neovascularization [20]. Neoangiogenesis is a vessels' principal response to inflammation. Recently, a relation between pulmonary fibrosis and wound healing and tumorogenesis characterized by neovascularization on the basis of existing vessels was emphasized [21]. Angiogenesis participates in the pathogenesis of idiopathic pulmonary fibrosis [8]. A prominent role of angiogenesis in the pathogenesis of other ILD, such as granulomatosis diseases, fibrosis and vasculitis has been also suggested [10-12, 22]. We do confirm this statement. The existence of neovascularization in IPF was originally identified by Turner-Warwick [23] who demonstrated the existence of anastomoses between the systemic and pulmonary microvasculature and presented evidence of extensive vascular remodeling in the areas of fibrosis. Previously, an increased angiogenic activity in the lung tissue of IPF [24] and COP [25] has been demonstrated. In the present study an increase in the angiogenic activity of sera from IPF and COP patients was observed. Keane et al. [26] have also demonstrated an increased angiogenic activity in experimental drug induced pulmonary fibrosis. Our results did not confirm proangiogenic activity of DLD patients' sera. However, we examined patients in inactive phase of the disease, not exposed to drugs. Although there is a great deal of evidence confirming the role of neovascularization in sarcoidosis, little is known about angiogenesis in hypersensitivity pneumonitis. An increase in a vascular endothelial growth factor (VEGF) level in serum in sarcoid granulomas and alveolar macrophages of patients with HP has been observed [27]. VEGF stimulates the degradation of extracellular matrix by metalloproteinases and may play a role in the pathogenesis of HP [28]. The angiogenic activity was demonstrated in lesions in collagen diseases [29]. Strong proangiogenic activity of 
sera from patients with collagen diseases with pulmonary manifestation was demonstrated in the present study. Little is known about angiogenesis in systemic vasculitis such as Wegener's granulomatosis. VEGF level can be raised in WG patients compared with normal controls [30]. We demonstrated that the angiogenic activity of sera from WG patients was similar to the serum activity in CD patients. There is also limited data on angiogenesis in lesions from patients with pneumoconiosis and with pulmonary Langerhans cell histiocytosis. In a group of patients with silicosis the increase in serum angiogenic activity was observed. By contrast, sera from patients with pulmonary Langerhans cell histiocytosis showed an inhibiting effect on angiogenesis. A similar antiangiogenic effect was also observed for sera from SCL patients. The present study confirms the presence of factors modulating angiogenesis in sera from patients with ILD, but indicates their various effects in different diseases both inhibiting or stimulating neovascularization.

Previous studies have focused on the role of angiogenesis in the pathogenesis of ILD, mostly on the cellular level. It is unclear whether angiogenic factors present in sera correlate with clinical symptoms and radiological changes in ILD patients. The evaluation of a relation between serum angiogenic activity and radiological changes is a difficult task, because the character of radiological changes widely differs. Previously, a correlation between serum VEGF level and a HRCT fibrosis score has been described [8]. A vascular supply to metastatic lung tumors is more variable, and there is a good radiographic evidence of angiogenesis in pulmonary circulation that supplies tumors [31]. In the present study, a significantly lower angiogenic activity of sera from patients with fibrotic radiological changes, compared with the activity in a group of patients with nodular pulmonary changes or mediastinal lymphatic changes, was demonstrated. Previously, we demonstrated a higher angiogenic activity of sera from patients in radiological stage II in comparison with stage I and III [10]. Stage II is characterized by an active inflammatory process with dominant nodular changes in the lungs and enlargement of thoracic lymphatic nodes. In stage III of sarcoidosis, there is an increase in fibrotic lung changes, while granulomas in mediastinal or hilar lymphatic nodes disappear. The results are convergent with observations Renzoni et al. [7] that angiogenesis may occur at earlier stages of the development of pulmonary fibrosis. However, pulmonary fibrosis is accompanied by a reduction in total vascular area and vascular density. That may explain why in patients with radiological end-stage fibrotic pulmonary changes a reduced angiogenic activity of sera was observed compared with the angiogenic activity of sera collected from patients with inflammatory nodular changes in the lungs and thoracic lymphatic nodes.

Serum angiogenic activity does not depend on age, sex, smoking habits, and the presence of clinical symptoms. Differences in serum angiogenic activity of the examined patients correlated with the severity of dyspnea. Patients with ILD and mild or moderate dyspnea showed a significantly reduced serum angiogenic activity compared with patients with severe dyspnea. The meaning of this observation is not known, as is not known the role angiogenesis plays in the pathogenesis of ILD. The hypervascularity observed in fibrotic areas may play a role in the regeneration of alveolar septa damaged by the fibrotic process and may be a compensatory response to decreased vascularity seen within fibroblastic foci. The other theory suggests that newly formed microvessels enhance the exaggerated and dysregulated extracellular matrix deposition, support fibroproliferation, and inhibit normal epithelial repair mechanisms [21]. Perhaps the role of angiogenesis in the pathologic process of pulmonary fibrosis is overestimated and that aberrant vascular remodeling is just a bystander, or a consequence of fibrogenesis. There are a number of possible explanations of the links between clinical and radiological changes and angiogenic activity of ILD patients' sera. Therefore, further research is needed to explain what effects neovascularization has on clinical course and on functional and structural respiratory disturbances in ILD. A limitation of the present study also is that it did not determine the serum factors modulating the angiogenic activity in ILD patients. We believe, however, we have shown that sera from ILD patients constitute a source of mediators modulating angiogenesis, but the pattern of reaction is different in various diseases.

Acknowledgments: Supported by an internal grant of the Institute of Tuberculosis and Lung Diseases in Warsaw, Poland.

Conflicts of interest: No conflicts of interest were declared by the authors in relation to this work.

\section{REFERENCES}

[1] Ryan HE, Lo J, Johnson RS. HIF-1 is required for solid tumor formation and embryonic vascularization. EMBO J 1998; 17: 3005-15.

[2] Tonnesen MG, Feng X, Clark RA. Angiogenesis in wound healing. J Investig Dermatol Symp Proc 2000; 5: 40-6.

[3] Jackson JR, Seed MP, Kircher CH, Willoughby DA, Winkler JD. The codependence of angiogenesis and chronic inflammation. FASEB J 1997; 11: 457-65.

[4] Carmeliet P, Jain RK. Angiogenesis in cancer and other diseases. Nature 2000; 407: 249-57.

[5] Klagsbrun M, Moses MA. Molecular angiogenesis. Chem Biol 1999; 6: R217-R24.

[6] Hanahan D, Folkman J. Patterns and emerging mechanisms of the angiogenic switch during tumorigenesis. Cell 1996; 86: 353-64.

[7] Renzoni EA, Walsh DA, Salmon M, Sestini P, Nicholson AG, Veeraraghavan S, Bishop AE, Romanska HM, Pantelidis $\mathrm{P}$, Black CM, du Bois RM. Interstitial vascularity in fibrosing alveolitis. Am J Respir Crit Care Med 2003; 167: 438-43.

[8] Simler NR, Brenchley PE, Horrocks AW, Greaves SM, Hasleton PS, Egan JJ. Angiogenic cytokines in patients with idiopathic interstitial pneumonia. Thorax 2004; 59: $581-5$.

[9] Strieter RM, Belperio JA, Keane MP. CXC chemokines in vascular remodeling related to pulmonary fibrosis. Am J Respir Cell Mol Biol 2003; 29 Suppl 3: S67-S9.

[10] Zielonka TM, Demkow U, Bialas B, Filewska M, Zycinska K, Radzikowska E, Szopinski J, SkopinskaRózewska E. Modulatory effect of sera from sarcoidosis 
patients on mononuclear cells-induced angiogenesis. J Physiol Pharmacol 2007; 58 Suppl 5: 753-66.

[11] Zielonka TM, Demkow U, Filewska M, Bialas-Chromiec B, Zycinska K, Szopinski J, Remiszewski P, Rowinska-Zakrzewska E, Wardyn KA, Skopinska-Rozewska E. Modulatory effect of sera from patients with various types of pulmonary fibrosis on mononuclear cells-induced angiogenesis in relation to pulmonary function. J Physiol Pharmacol 2008; 59 Suppl 6: 771-9.

[12] Zielonka TM, Demkow U, Filewska M, Bialas-Chromiec B, Zycinska K, Radzikowska E, Korzeniowska M, Wardyn KA, Kus J, Skopinska-Rozewska E. Angiogenic activity of sera from silicosis and pulmonary Langerhans cell histiocytosis patients in relation to lung function tests. J Physiol Pharmacol 2008; 59 Suppl 6: 781-9.

[13] Wells AU, Rubens MB, du Bois RM, Hansell DM. Functional impairment in fibrosing alveolitis: relationship to reversible disease on thin section computed tomography. Eur Respir J 1997; 10: 280-5.

[14] ATS/ERS/WASOG. Statement on sarcoidosis. Sarcoidosis Vasc Diffuse Lung Dis 1999; 16: 149-73.

[15] American Thoracic Society. Idiopathic pulmonary fibrosis: diagnosis and treatment. International consensus statement. American Thoracic Society (ATS) and the European Respiratory Society (ERS). Am J Respir Crit Care Med 2000; 161: 646-64.

[16] Lacasse Y, Selman M, Costabel U, Dalphin JC, Ando M, Morell F, Erkinjuntti-Pekkanen R, Muller N, Colby TV, Schuyler M, Cormier Y, HP Study Group. Clinical diagnosis of hypersensitivity pneumonitis. Am J Respir Crit Care Med 2003; 168: 952-8.

[17] American Thoracic Society/European Respiratory Society. International Multidisciplinary Consensus Classification of the Idiopathic Interstitial Pneumonias. Am J Respir Crit Care Med 2002; 165: 277-304.

[18] Sidky YA, Auerbach R. Lymphocyte-induced angiogenesis: a quantitative and sensitive assay for the graft-versushost reaction. J Exp Med 1975; 141: 1084-100.

[19] Demedts M, Wells AU, Anto JM, Costabel U, Hubbard R, Cullinan P, Slabbynck H, Rizzato G, Poletti V, Verbeken EK, Thomeer MJ, Kokarinen J, Dalphin JC, Taylor AN. Interstitial lung diseases: an epidemiological overview. Eur Respir J 2001; 18 Suppl 32: 2s-16s.

[20] Humar R, Kiefer FN, Berns H, Resink JT, Battegay EJ. Hypoxia enhances vascular cell proliferation and angiogenesis in vitro via rapamycin (mTOR) - dependent signaling. FASEB J 2002; 16: 771-80.

[21] Selman, M, King TE, Pardo A. Idiopathic pulmonary fibrosis: prevailing and evolving hypotheses about its pathogenesis and implications for therapy. Ann Intern Med 2001; 134: 136-51.
[22] Tzouvelekis A, Anevlavis S, Bouros D. Angiogenesis in interstitial lung diseases: a pathogenetic hallmark or a bystander? Respir Res DOI: 10.1186/1465-9921-7-82, May 2006.

[23] Turner-Warwick M. Precapillary systemic-pulmonary anastomoses. Thorax 1963; 18: 225-37.

[24] Keane MP, Arenberg DA, Lynch JP III, Whyte RI, Iannettoni MD, Burdick MD, Wilke CA, Morris SB, Glass MC, DiGiovine B. The CXC chemokines, IL-8 and IP-10, regulate angiogenic activity in idiopathic pulmonary fibrosis. J Immunol 1997; 159: 1437-43.

[25] Lappi-Blanco E, Soini Y, Kinnula V, Paakko P. VEGF and bFGF are highly expressed in intraluminal fibromyxoid lesions in bronchiolitis obliterans organizing pneumonia. J Pathol 2002; 196: 220-27.

[26] Keane MP, Belperio JA, Moore TA Moore BB, Arenberg DA, Smith RE, Burdick MD, Kunkel SL, Strieter RM. Neutralization of the CXC chemokine, macrophage inflammatory protein-2, attenuates bleomycin-induced pulmonary fibrosis. J Immunol 1999; 162: 5511-8.

[27] Navarro C, Ruiz V, Gaxiola M, Carrillo G, Selman M. Angiogenesis in hypersensitivity pneumonitis. Arch Physiol Biochem 2003; 111: 365-8.

[28] Pardo A, Barrios R, Gaxiola M, Segura-Valdez L, Carrillo G, Estrada A, Mejia M, Selman M. Increase of lung neutrophils in hypersensitivity pneumonitis is associated with lung fibrosis. Am J Respir Crit Care Med 2000; 161: 1698 1704.

[29] Szekanecz Z, Szegedi G, Koch AE. Angiogenesis in rheumatoid arthritis. J Invest Med 1998; 46: 27-41.

[30] Li CG, Reynolds I, Ponting JM, Holt PJ, Hillarby MC, Kumar S. Serum levels of vascular endothelial growth factor (VEGF) are markedly elevated in patients with Wegener's granulomatosis. Br J Rheumatol 1998; 37: 1303-6.

[31] Mitzner W, Lee W, Georgakopoulos D, Wagner E. Angiogenesis in the mouse lung. Am J Pathol 2000; 157: 93-101.

Author's address:

Tadeusz Maria Zielonka M.D., Ph.D.

Department of Family Medicine

Warsaw Medical University

Banacha St. 1a

02-097 Warsaw

Poland

Phone/Fax: +48 223186325

E-mail: tmzielonka@wp.pl 